\title{
Effect of 2 types of resistant starches on the quality of yogurt
}

\author{
Jun He, Yumei Han, ${ }^{*}$ Min Liu, Yanan Wang, Yang Yang, and Xujin Yang \\ College of Food Science and Engineering, Inner Mongolia Agricultural University, Huhhot 010018, China
}

\section{ABSTRACT}

This study investigated the effects of resistant starch (RS) 2 (a high-amylose corn starch) and RS3 (physically modified corn starch) on yogurt quality. Yogurt containing Bifidobacterium BB-12 was treated with RS2 or RS3 to a final concentration of $1.5 \%$, with the control group receiving $1.5 \%$ (wt/wt) of sucrose. Multispeckle diffusing wave spectroscopy and scanning electron microscopy were used to investigate the effect of the resistant starches on the gelation process and microstructure of yogurt. The quality of the yogurt treatments was evaluated using viable counts of Bifidobacterium BB-12 and all viable cells, titratable acidity, amount of whey separation, and viscosity during storage. The resistant starches affected the progress of gelation and microstructure and decreased the decline of viable counts of Lactobacillus. Notably, RS3 effectively protected the Bifidobacterium BB-12, increased the viscosity, and decreased titratable acidity. Our results suggested that RS could improve the quality of yogurt and have a more probiotic effect. Further studies could lead to optimization in yogurt processing by mixing these 2 types of RS to determine their best usage and explore their interactions with proteins.

Key words: resistant starch, yogurt, quality

\section{INTRODUCTION}

Resistant starch (RS) is a form of dietary fiber found naturally in many starchy foods. It escapes digestion and absorption in the human small intestine and is fermented in the large intestine by probiotic bacterial providing beneficial effects (Raigond et al., 2015). The $\mathrm{RS}$ is classified into 5 subtypes: RS1 (physically inaccessible to digestion), RS2 (native starch granules), RS3 (physically modified starch), RS4 (chemically modified starch), and RS5 (amylose-lipid complexes; Raigond et al., 2015). With stable structure and acid or heating resistance, RS2 and RS3 are more common in

Received August 16, 2018.

Accepted January 10, 2019.

*Corresponding author: zwzyly403@163.com research on application of RS but may differ in application due to their particular characteristics (Ratnayake and Jackson, 2008).

Yogurt is formed by the fermentation of lactose to lactic acid by the starter bacteria Lactobacillus delbrueckii ssp. bulgaricus and Streptococcus thermophilus (Morelli, 2014). There is a growing need to produce fermented products that do not whey-off during storage with the use of fewer chemical additives or additives that are familiar to the public. In the dairy industry, starch or modified starch is widely used mainly due to their properties of stabilization, emulsification, and structural formation (Kett et al., 2013; Rezaei et al., 2015). The starches RS2 and RS3 could be good choices due to their stable structure during thermal sterilization or acidification in the process and storage of yogurt. Despite the utilization of RS in yogurt, there has been little comparative study of RS2 and RS3 in yogurt systems. Probiotic yogurts, being widely consumed, are prepared with probiotics, which are viable microorganisms that are healthy for the host, and can be fermented separately or mixed with ordinary starter bacteria during yogurt production (Aryana and Olson, 2017). There is a positive health image associated with probiotic products, and maintaining viability of probiotic cultures in yogurt during storage is important for providing efficacious probiotic yogurt (Hill et al., 2014). Bifidobacteria grow poorly in milk compared with yogurt starter, but some prebiotics can protect them (Rosburg et al., 2010; Prasanna et al., 2014). It is accepted that the prebiotic substances can enhance the viability of maintaining Bifidobacterium above the minimum therapeutic level of $10^{7} \mathrm{cfu} / \mathrm{g}$ to achieve the claimed health benefits (Senaka Ranadheera et al., 2012). The RS are regarded as a kind of prebiotic that can maintain a certain level of probiotics in yogurt under extreme conditions, but systematic research of interactions of RS2 and RS3 with bifidobacteria in yogurt is lacking (Etchepare et al., 2016; Zheng et al., 2016).

The aim of this work was to study the effect of RS2 and RS3 on the gel structure of yogurt containing bifidobacteria based on multispeckle diffusing wave spectroscopy, a light-scattering technique that uses the short-term dynamics of scattering particles to detect 
gel formation from the changes in elastic index (EI), macroscopic viscosity index (MVI), and solid-liquid balance (SLB; Rohart et al., 2016) and by examining microstructure with scanning electron microscopy. Viable counts of Bifidobacterium BB-12 and all lactobacilli, titratable acidity, amount of whey separation, and viscosity of yogurt during storage were also assessed. The yogurt containing sucrose was the control sample used to judge the effect of RS as carbon sources on the yogurt. The information gained should pave the way for better application of RS in yogurt.

\section{MATERIALS AND METHODS}

\section{Materials}

The main instruments were a Rheolaser Master (Formulaction, Toulouse, France), a scanning electron microscope (Pro; Phenom, Shanghai, China), a rotational viscosimeter (DV-2 Pro; Nirun, Shanghai, China), and a high-pressure homogenizer (Donghua, Shanghai, China). The RS3 resistant corn starch was obtained from Yuanye Biotechnology (Shanghai, China), and RS2, a high- amylose corn starch, was obtained from Ingredion Inc. (Westchester, IL). Whole milk powder was obtained from Fonterra Co-operative Group (Auckland, New Zealand), yogurt starter species YF-L812 and Bifidobacterium BB-12 from Chr. Hansen (Hørsholm, Demark), anaerobic gas bag from Mitsubishi Gas Chemical (Tokyo, Japan), De Man, Rogosa and Sharpe (MRS) solid medium from HuanKai Biotechnology (Guangzhou, China), and lithium mupirocin from Haibo Biotechnology (Changsha, China).

\section{Set Yogurt Preparation}

Skim milk was prepared from milk powder with a concentration of $11.5 \%$ (wt/wt), and RS2, RS3, or sucrose were added to a final concentration of $1.5 \%$ (Costa et al., 2015). Then, the samples were mixed thoroughly, filtered, and preheated to $60^{\circ} \mathrm{C}$ for $30 \mathrm{~min}$. After homogenization at 20 to $25 \mathrm{MPa}$, the mixture was heat treated at $95^{\circ} \mathrm{C}$ for 5 min to pasteurize and then cooled to $40^{\circ} \mathrm{C}$. Incubation of the yogurt samples was carried out at 42 to $43^{\circ} \mathrm{C}$ for about $4.5 \mathrm{~h}$, when the $\mathrm{pH}$ value reached 4.5. The concentration of the starter bacterial culture was $9 \times 10^{8} \mathrm{cfu} / \mathrm{g}$, and the concentration for Bifidobacterium BB-12 was $6 \times 10^{8} \mathrm{cfu} / \mathrm{g}$. Finally, the prepared yogurt samples were kept at $4^{\circ} \mathrm{C}$ for $1 \mathrm{~d}$ of postfermentation and then stored at $4^{\circ} \mathrm{C}$. Samples were analyzed at $0,1,3,7,14$, and $21 \mathrm{~d}$ of storage.

\section{Rheological Measurements During Fermentation}

Milk samples (20 $\mathrm{mL}$ for Rheolaser Master) were poured into glass tubes (inner diameter of $27.5 \mathrm{~mm}$ for Rheolaser Master; Rohart et al., 2016). The Rheolaser Master apparatus was equilibrated at $43^{\circ} \mathrm{C}$. Sample cells were immediately placed in the apparatus, and the milk was left to acidify until reaching $\mathrm{pH} 4.5$ and then stored at $4^{\circ} \mathrm{C}$ for $1 \mathrm{~d}$ before further measurement. Measurements were taken every $1 \mathrm{~min}$. Collection and analysis of original data were performed by the software attached to the instrument and directly provided the rheological parameters EI, MVI, and SLB.

\section{Microstructure Observation by Scanning Electron Microscopy}

The evaluation of microstructure of yogurts can be favorable for analyzing its physical properties and quality. The scanning electron microscopy was carried out according to Cui et al. (2014) and Bakirci et al. (2017) with some modifications. Preparation of samples was as follows: the sample after postfermentation was set in a small glass with a thin coating layer, frozen in liquid nitrogen, and then rapidly dried in a vacuum freeze dryer. The yogurt samples were lyophilized and then mounted on aluminum stubs. The samples were viewed and photographed at an accelerating voltage of $10 \mathrm{kV}$ and magnification of $5,000 \times$. All measurements were done for rheological analysis at least 3 times.

\section{Measurement of Titratable Acidity}

Total titratable acidity was determined in 10-g samples in $90 \mathrm{~mL}$ of distilled water and expressed as the volume $(\mathrm{mL})$ amount of $0.1 \mathrm{~mol} / \mathrm{L} \mathrm{NaOH}$ to obtain $\mathrm{pH}$ 8.3. The titratable acidity was calculated based on lactic acid being the predominant acid and was expressed as $1^{\circ} \mathrm{T}=100 \mathrm{mg}$ of lactic acid/L (Jana et al., 2015). All determinations were repeated at least 3 times.

\section{Measurement of Viscosity}

Viscosity of yogurt was determined according to Jiaojiao et al. (2015) with some modifications. Measurements were carried out at $25^{\circ} \mathrm{C}$ with a viscometer (Nirun) with spindle (no. 21). All measurements were recorded at $20 \mathrm{rpm}$. The yogurt was gently stirred for $20 \mathrm{~s}$ (20-s continuous sweeps) before analysis. All determinations were repeated at least 3 times. 


\section{Measurement of Whey Separation}

The amount of whey separation was measured using the method of Lobato-Calleros et al. (2014). Ten grams of yogurt $\left(4 \pm 1^{\circ} \mathrm{C}\right)$ was placed in tubes and centrifuged at $2,227 \times g$ for $10 \mathrm{~min}$ at $4 \pm 1^{\circ} \mathrm{C}$. The clear supernatant was poured off, weighed, and expressed as percentage weight relative to original weight of yogurt. Syneresis analyses were carried out in triplicate.

\section{Viable Cell Counts}

Counts of viable bacteria and Bifidobacterium BB12 were performed according to a previously published method (Buriti et al., 2010; Etchepare et al., 2016) with modifications. The total viable counts of the prepared subsequent serial dilutions were enumerated using the roll-tube technique, and serial dilutions were transferred to sterile Petri plates containing MRS agar for total viable counts and MRS agar with lithium mupirocin for Bifidobacterium BB-12 counts. In triplicate, plates were anaerobically incubated at $37 \pm 1^{\circ} \mathrm{C}$ for 48 $\mathrm{h}$ in jars with a gas generator envelope (Anaero Pack, Mitsubishi Gas Chemical). Colonies on plates containing 30 to 300 colonies were counted and expressed as colony-forming units per gram of the yogurt product.

\section{Data Analysis}

Results were submitted to ANOVA procedures using SAS 9.3 (SAS Institute Inc., Cary, NC). Statistical graphs were constructed using Origin 9.1 (Origin Lab, Northhampton, MA).

\section{RESULTS AND DISCUSSION}

\section{Rheological Characteristics}

Casein gel, the main constituent of yogurt structure, is dynamic by nature, and excessive rearrangements of particles making up the gel network before and during gelation are responsible for the fermentation process (Titapiccolo et al., 2011). In this study, the evolution of parameters EI, MVI, and SLB by multispeckle diffusing wave spectroscopy was used to monitor the gelation process of yogurt with or without addition of different RS and sucrose. The EI is directly proportional to the elastic modulus as a function of time, and the MVI is directly proportional to the viscosity modulus as a function of time (Figures 1 and 2; Rohart et al., 2016). The SLB is directly proportional to the viscoelastic properties of the product and gives an indication of the ratio between solid-like and liquid-like behavior of the

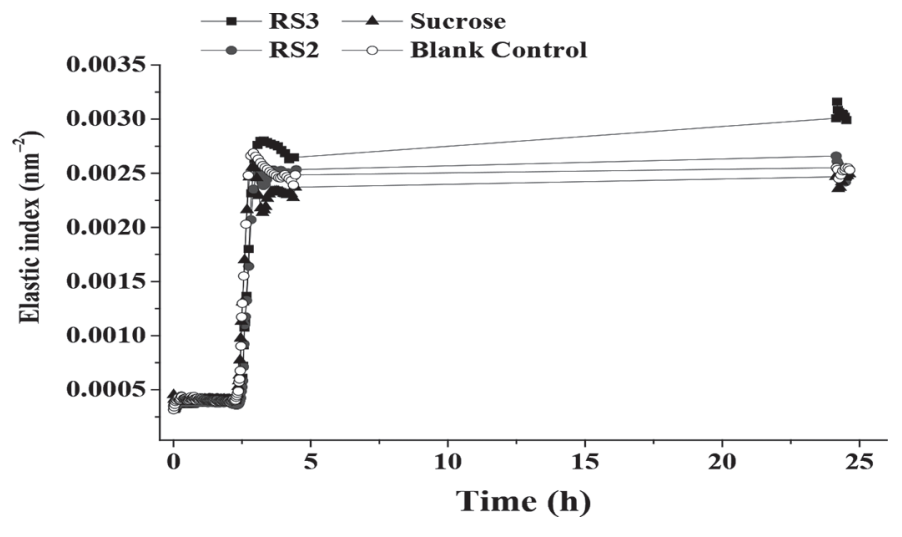

Figure 1. Elastic index of yogurt during fermentation. RS2 = resistant starch 2 (native starch granules); RS3 = resistant starch 3 (physically modified starch).

product as a function of time (Figure 3). The yogurt was elastic or solid-like for an SLB of 0 to 0.5 and viscous or liquid-like for an SLB of 0.5 to 1 (Rohart et al., 2016).

The EI or MVI of all samples showed the same pattern of changes (Figures 1-3). Initially, the EI of all set yogurts remained stable during 0 to $2.5 \mathrm{~h}$ for casein without forming a gel structure but began to dissociate with microbial acidification. Also, the MVI of all set yogurts remained at one level with a small fluctuation during the same period; however, the MVI of the blank control sample was the lowest, meaning that the additives increased the viscosity of milk before it turned into gel. In addition, the SLB of all samples quickly increased to a peak from a relatively low initial point and then decreased with fluctuations before $2.5 \mathrm{~h}$. The

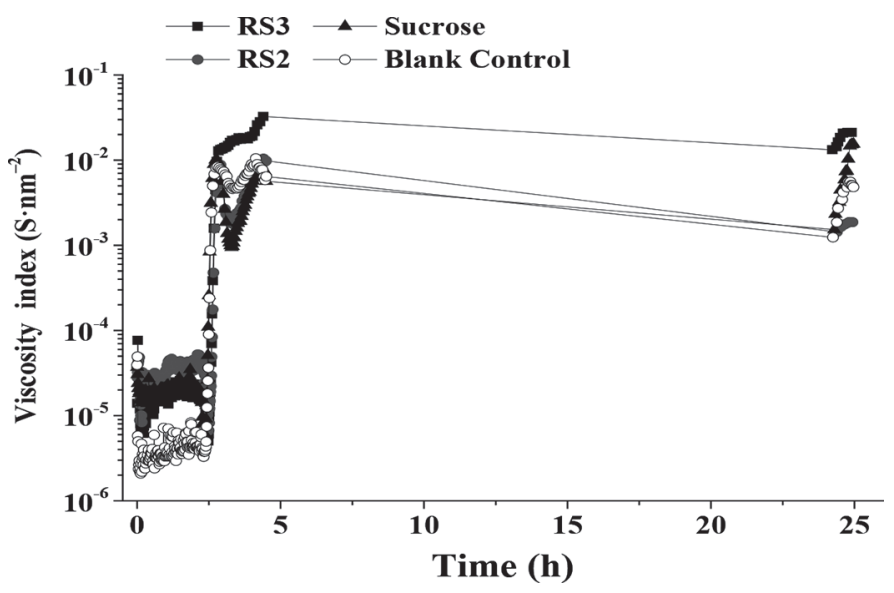

Figure 2. Viscosity index of yogurt during fermentation. RS2 = resistant starch 2 (native starch granules); RS3 = resistant starch 3 (physically modified starch). 
SLB of the blank control was more than double that of other samples because the additives changed the water activity or hydrophobic attraction with casein such that the particles had limited movement with the lower SLB. All yogurts presented more solid characteristics at first, as their SLB in the initial fermentation were lower than in later fermentation (Sharafbafi et al., 2014).

All samples reached a gel point at about $150 \mathrm{~min}$. Therefore, RS may not have affected fermentation time, and the fermentation time is mainly relevant to the acid kinetic of starter bacteria (Marafon et al., 2011). Thus, RS might not affect the metabolism of starter bacteria in the period. When the samples reached the gel point, the EI and MVI of all samples increased to their highest levels, with casein micelle aggregation and formation of gel structure (Lazaridou et al., 2014). Additionally, yogurt with RS3 had higher EI and MVI but lower SLB than the other samples, showing that RS3 contributed strength to the gel structure.

In the next stage, starter bacteria increased acidity, which caused casein to aggregate (Pang et al., 2016). The EI of all samples decreased slightly and the MVI fluctuated, which could be explained by the bacteria producing acid, resulting in solubilization of colloidal calcium phosphate within and between casein micelles, thus weakening the network structure (Lazaridou et al., 2014). As the isoelectric point of caseins is approached, the repulsions between casein particles decrease and the hydrophobic interactions are enhanced; rapid aggregation causes extensive rearrangements in the protein network, incorporation of additional protein

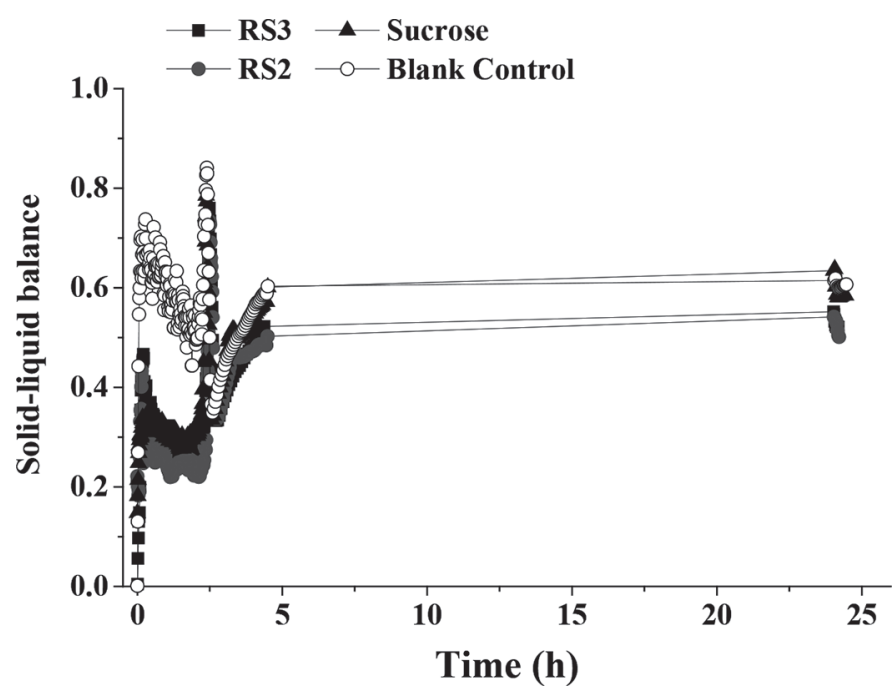

Figure 3. Solid-liquid balance of yogurt during fermentation. RS2 $=$ resistant starch 2 (native starch granules); RS3 = resistant starch 3 (physically modified starch). clusters into the gel network, or both (Lazaridou et al., 2014). There were continuous increases of EI up to a maximum value, and the MVI remained steady after some fluctuation. After that, the casein micelles rebuilt the network structure, the SLB increased and EI declined to a steady state. Finally, yogurt containing RS had higher elastic modulus and loss modulus than the sucrose and blank controls. However, RS may have different combination forms in yogurt because their EI and MVI values differed, and yogurt with RS3 was more solid, with a relatively compact structure.

\section{Scanning Electron Microscopy}

There were pronounced microstructural differences among the yogurts with or without RS2, RS3, and sucrose as visualized by scanning electron microscopy (Figure 4). Microstructure of yogurt comprises a 3-dimensional network of casein aggregates in that their globular shape is still discernible and interspersed by voids containing serum. The lack of the cell of bacteria in yogurt may have been due to the vacuum freezedrying, which might have led to bursting of lactic acid bacteria. The different behavior of RS (Figure 4a and b) compared with sucrose (Figure 4c) on yogurt gel texture was due both to the specific properties of these substances and to their different ways of interfering with the formation of the protein network (Salvatore et al., 2014). Similar to other chemical-modified starches (Cui et al., 2014), RS played an important role in the development of yogurt texture, distributed within the casein matrix.

The yogurt containing RS3 (Figure 4a) was characterized by a very compact structure, comprising a network of casein micelles and fat. This was in agreement with previous studies of starch-based dairy (Kett et al., 2013; Cui et al., 2014). There was almost a total lack of pores compared with the blank control, and the gel network of yogurt containing sucrose had dense colloidal particles, with the network clearly displaced. The RS3 appeared as structures embedded in the gel system, and the casein micellar or colloidal particles shown as debris might have been fixed by it. Additionally, the casein micelles seemed to be larger in the RS3 compared with other samples, which meant that RS3 tended to make the casein aggregate. The images showed RS2 inserted between the protein aggregates, forming a fibrous structure and decreasing the number of voids but increasing their diameter; this resembled the microstructure of yogurt containing cross-linked waxy maize starch (Azim et al., 2010). In contrast to RS3, yogurt with RS2 had a relatively loose, spongy structure with many holes, with a block inserted between the protein aggregates to 
form a fibrous structure and the starch forming a more continuous and compact network structure. The low numbers of pores indicated a stronger gel and strong interactions between casein particles (Marafon et al., 2011), which was consistent with the RS3 treatment having more elastic and solid properties. Sucrose has good solubility and is easily utilized by microorganisms in yogurt (Costa et al., 2015). Thus, the starter bacteria in yogurt with sucrose added could have greater metabolic efficiency and produce more exopolysaccharides, which filled holes and decreased the empty space in gel networks, demonstrating that the RS was not as fully utilized as sucrose was by lactic acid bacteria.

\section{Titratable Acidity}

Titratable acidity affects viability of probiotic bacteria during production or storage and affects the quality of fermented dairy products. Acid production during fermentation of lactose is the primary functional requirement for yogurt starter bacteria (Mishra and Mishra, 2013). During storage, the acid produced by bacteria could negatively affect yogurt quality, which was called the effect of postacidification (Mishra and Mishra, 2013; Liu et al., 2014). Thus, the amount of acid production can be used to indicate bacterial activity. Some prebiotic additives affect the sensory or acid production via changes of enzyme activity with hydration, and some cause bacteria to change their metabolism (Santos et al., 2015).

The titratable acidity of all samples increased progressively through storage from almost $60^{\circ} \mathrm{T}$ to $70^{\circ} \mathrm{T}$ (Figure 5). During the previous period of fermentation storage, the titratable acidity sharply increased due to the amount of lactic acid bacteria and their metabolic activity. After $3 \mathrm{~d}$, the titratable acidity continued to increase slightly. Significant differences $(P<0.05)$ were found in titratable acidity of yogurts among the treatments. Yogurt with RS3 had a lower titratable acidity than other samples based on multiple comparisons of means during the entire storage period. Compared with the blank control and sucrose, the RS could not increase the acidity of yogurt as a carbon source for bacteria such as sucrose, and RS might change the water activ-
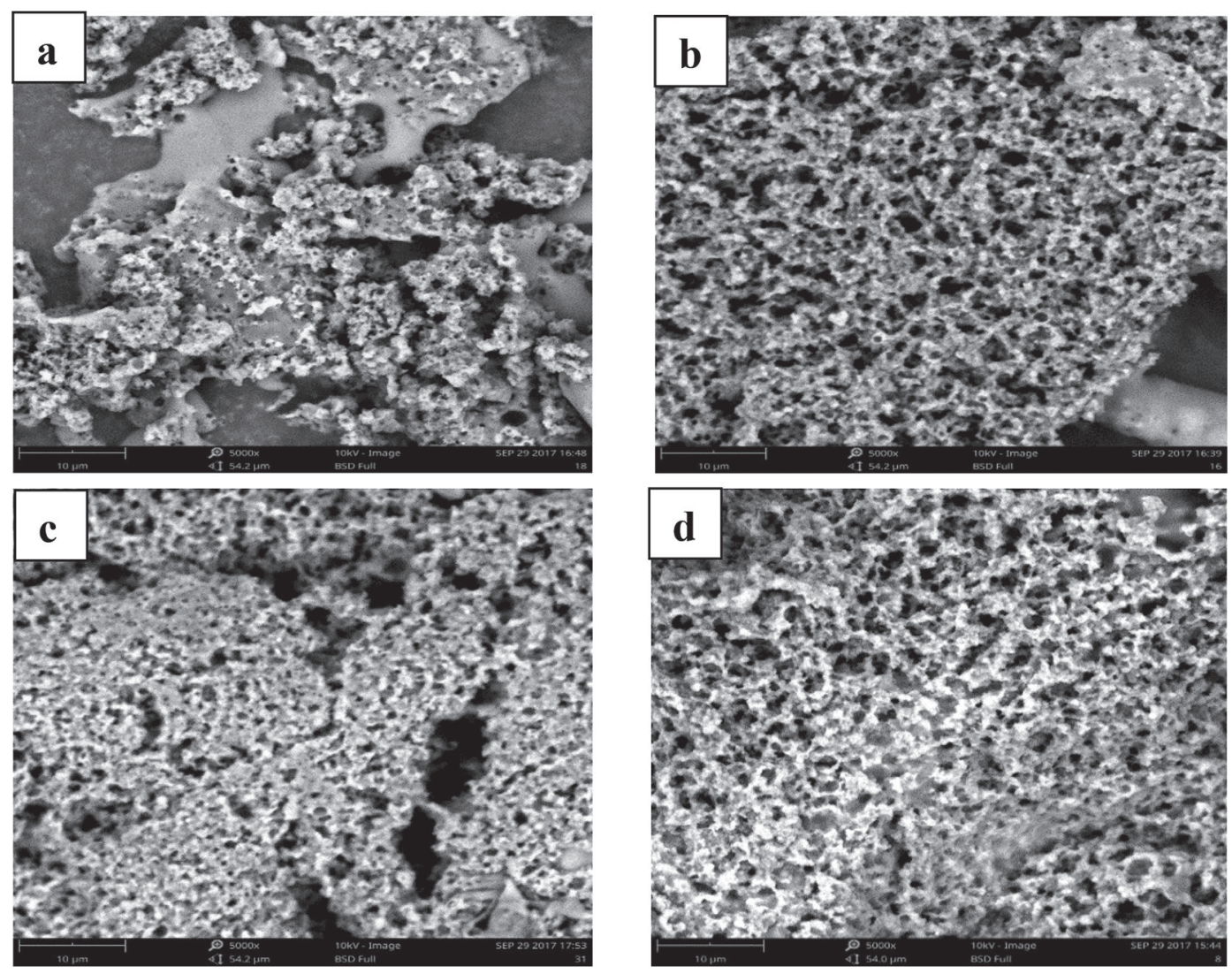

Figure 4. Scanning electron micrographs of the set yogurt: (a) 1.5\% (wt/wt) resistant starch 3 (RS3; physically modified starch); (b) $1.5 \%$ (wt/wt) resistant starch 2 (RS2; native starch granules); (c) 1.5\% (wt/wt) sucrose; and (d) blank control. Scale bar $=10 \mu \mathrm{m}$. 


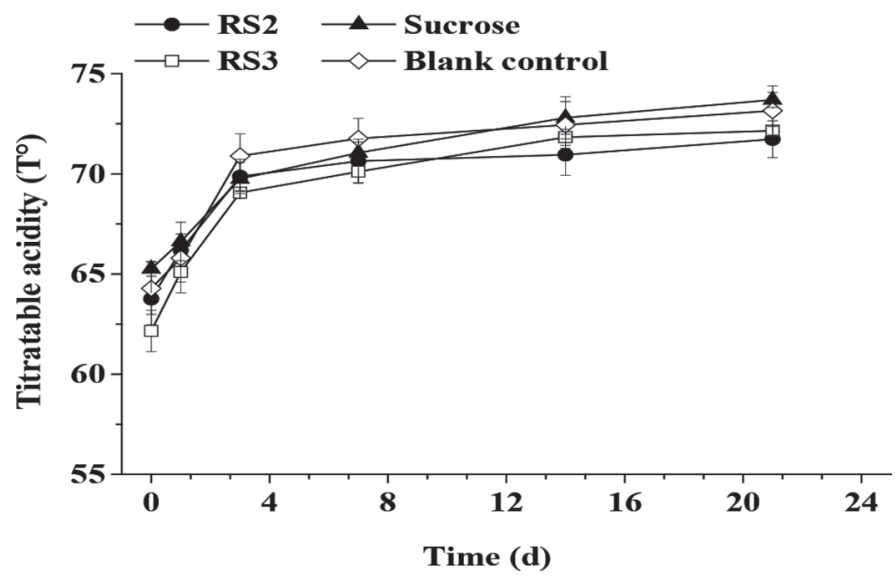

Figure 5. Titratable acidity of yogurt during storage. RS2 $=$ resistant starch 2 (native starch granules); RS3 = resistant starch 3 (physically modified starch). Error bars show SD of the results from at least 3 independent experiments.

ity to affect bacterial metabolism but could not utilize it (Costa et al., 2015).

\section{Viscosity}

Previous studies found that blends of starch and casein were synergistic in terms of viscosity formation, which could also form a gel, and the gel might be a product of particulate starch filling the protein network to make it more rigid (BeMiller and Whistler, 2009). During a certain period of fermentation or storage, hydration of components is completed, meaning that increased aging time increases viscosity, as was found. However, viscosity of all samples declined after a relatively long storage time for the unstable structure in yogurts (Etchepare et al., 2016).

Addition of RS2 and RS3 strongly increased yogurt viscosity (Figure 6). A similar but nonidentical result was obtained for MVI above because MVI was determined from particle movement and viscosity was measured based on torque drive at a certain frequency of shear. At storage of 1 and $3 \mathrm{~d}$, the sucrose treatment was more viscous than RS2, and the bacteria in yogurts containing sucrose may have produced more exopolysaccharide, which increased viscosity and acidity (Mishra and Mishra, 2013). However, yogurts containing RS2 or RS3 were more viscous than others, not by affecting bacterial metabolism with the lower acidity but due to increased hardness of the protein matrix (Figure 3). The effect of addition of RS on viscosity was presumably the result of water retention by soluble fiber and an increase in serum concentration. The RS3 resulted in greater increases in viscosity compared with RS2 due its different way of combining with the gel network or affecting casein in accordance with the scanning electron microscopy results.

\section{Whey Separation}

Whey separation is related to instability of the gel network, and the amount of serum separation affects yogurt quality during storage (Pang et al., 2016). High-speed centrifugation can measure the yogurt's whey-holding capacity and gel rigidity under relatively high forces (Lobato-Calleros et al., 2014). Consistent with the SLB and EI, the RS treatment resulted in yogurt with a strong network and lower amounts of whey separation $(P<0.05)$ during the whole process of fermentation and storage. Especially in the later storage period, the amounts of serum yogurts containing $\mathrm{RS}$ were within 0.3 , whereas the blank control grew to 0.4 and the sucrose treatment declined to 0.35 .

Many previous studies have shown that when 2 complex systems such as starch and milk ingredients are mixed, the stability of the mixed systems depends mainly on their physicochemical properties (Pang et al., 2016). The average amount of whey separation of yogurt containing RS2 during the whole process of fermentation and storage was lower than that of yogurt containing RS3 (Figure 7). Thus, RS2 induced the gel network to hold more serum than RS3, especially during the end of storage, when the high titratable acidity led to weakness of casein micelle structure (Marafon et al., 2011). Similar to previous conclusions, it was reported that the long polysaccharide chain of inulin

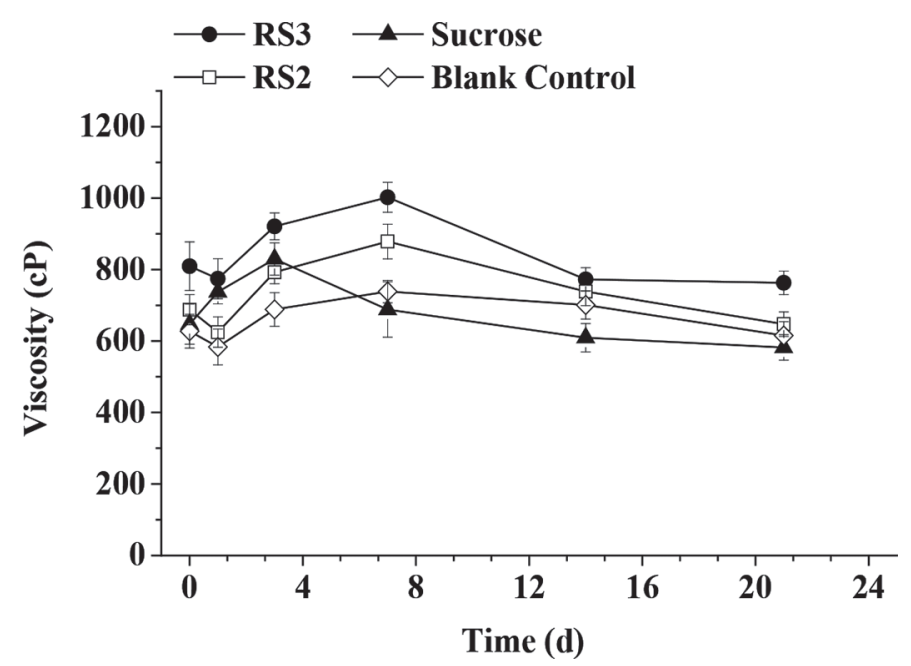

Figure 6. Viscosity of yogurt during storage. RS2 = resistant starch 2 (native starch granules); RS3 = resistant starch 3 (physically modified starch). Error bars show SD of the results from at least 3 independent experiments. 


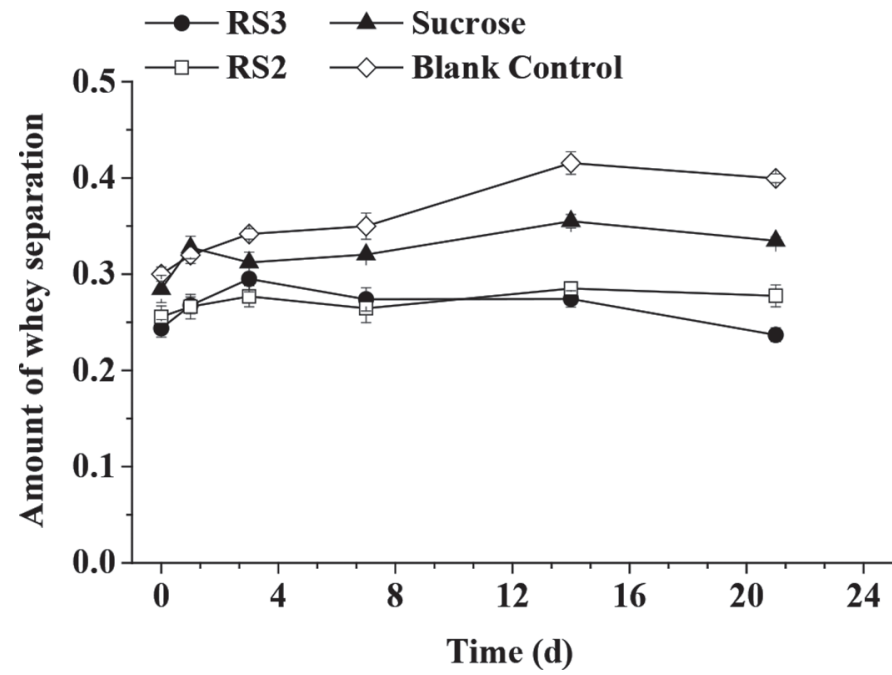

Figure 7. Amount of whey separation of yogurt during storage. RS2 = resistant starch 2 (native starch granules); RS3 = resistant starch 3 (physically modified starch). Error bars show SD of the results from at least 3 independent experiments.

might be dispersed among the casein micelles, interfering with formation of the protein matrix and therefore responsible for a softer gel network (Lobato-Calleros et al., 2014). Because RS2 and RS3 have different structures, this could lead to differences in formation of the protein matrix consistent with the microstructure shown by scanning electron microscopy (Ratnayake and Jackson, 2008).

\section{Counts of Yogurt Bacteria}

Viable counts of starter bacteria in yogurts during storage decreased sharply during 1 to $14 \mathrm{~d}$ (Figure 8a) and were corroborated by the results of titratable acidity related to microbial activity. After $21 \mathrm{~d}$ of storage, the counts were only 15 to $20 \%$ lower than the initial counts in all samples $(P<0.05)$. Compared with the concentration of starter bacterial culture before fermentation and the counts of all viable bacteria and Bifidobacterium BB-12 in yogurt, yogurt starter bacteria represented the majority of viable bacteria, suggesting that yogurt starter bacteria mostly affected the quality of yogurt during storage. The blank control had the lowest count and the yogurt-containing sucrose had the highest count in the mean value test during 0 to 21 d. This could be due to the effect of carbon sources, with the RS being difficult for bacteria to utilize during fermentation, and consistent with the fact that sucrose increased the titratable acidity faster than the RS treatments.

Storage significantly affected the counts of probiotic bacteria in yogurt $(P<0.01)$. All yogurt samples showed rapid declines in the numbers of Bifidobacterium BB-12 before $7 \mathrm{~d}$ of storage, and the amounts remained stable with slight changes after $14 \mathrm{~d}$. The Bifidobacterium BB12 did not grow and multiply during fermentation, for its viable counts in yogurt were lower than the amounts of inoculum, and the amount of inoculum was $6 \times 10^{8}$ $\mathrm{cfu} / \mathrm{g}$, which was more than in yogurt (Figure 8b). Thus, yogurt containing probiotics such as Bifidobacterium BB-12 needs protection to maintain a certain amount for beneficial function (Sendra et al., 2016). Previous studies have suggested that RS could form starch-containing microcapsules to protect probiotic bacteria in adverse conditions (Etchepare et al., 2016).

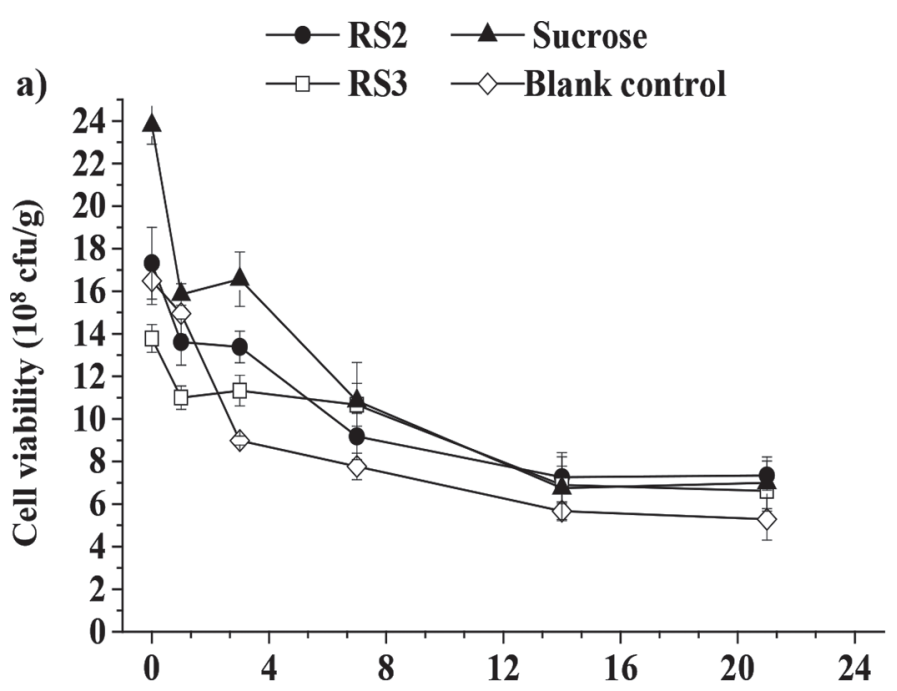

b)

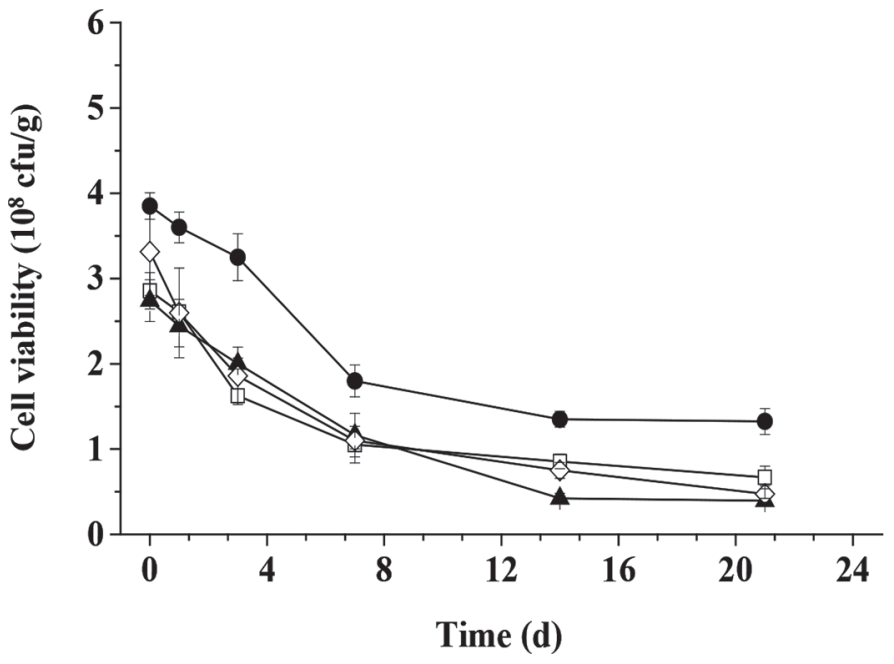

Figure 8. Cell viability of the set yogurt with (a) all viable bacteria and (b) Bifidobacterium BB-12. RS2 = resistant starch 2 (native starch granules); RS3 = resistant starch 3 (physically modified starch). Error bars show SD of the results from at least 3 independent experiments. 
However, in this study there were no microcapsules, and RS2 did not protect Bifidobacterium BB-12 as effectively as the sucrose treatment did. The RS3 treatment resulted in Bifidobacterium BB-12 remaining above $10^{8}$ $\mathrm{cfu} / \mathrm{g}$ during 0 to $21 \mathrm{~d}$, which was significantly higher $(P<0.05)$ than in the other treatments. Thus, RS3 could protect probiotics such as Bifidobacterium BB-12 in yogurt during storage.

\section{CONCLUSIONS}

Use of RS in yogurt samples was observed to improve qualitative characteristics. Serum separation, viscosity, flow behavior, viscoelastic properties, and titratable acidity of formulated samples containing the 2 RS showed significant differences compared with the control. These 2 RS altered the quality of yogurts due to their different effects on gel structure formation and not simply as carbon sources for lactic acid bacteria. The yogurt with RS2 induced the gel network to hold more serum. However, the yogurt with RS3 showed improved viscosity and was better at controlling postfermentation acidity in addition to protecting probiotic organisms, which will be helpful in strengthening probiotic function. Mixing these 2 types of RS for their best usage and interactions with protein for better gel structure, as well as using RS as prebiotics and probiotics in a yogurt system to provide a doubly healthful product, will be investigated in future studies.

\section{ACKNOWLEDGMENTS}

We are grateful to the Natural Science Fund of Inner Mongolia (grant no. 2016MS0539, Huhhot, China) and Technology Project Fund of Inner Mongolia (grant no. 201502094, Huhhot, China) for providing financial support, and the College of Food Science and Engineering, Inner Mongolia Agricultural University for providing laboratory facilities for this research work. We also thank International Science Editing (http:// www.internationalscienceediting.com) for editing this article.

\section{REFERENCES}

Aryana, K. J., and D. W. Olson. 2017. A 100-year review: Yogurt and other cultured dairy products. J. Dairy Sci. 100:9987-10013.

Azim, Z., M. Alexander, M. Koxholt, and M. Corredig. 2010. Influence of cross-linked waxy maize starch on the aggregation behavior of casein micelles during acid-induced gelation. Food Biophys. 5:227-237.

Bakirci, S., E. Dagdemir, O. S. Boran, and A. A. Hayaloglu. 2017. The effect of pumpkin fibre on quality and storage stability of reducedfat set-type yogurt. Int. J. Food Sci. Technol. 52:180-187.

BeMiller, J. N., and R. L. Whistler. 2009. Starch: Chemistry and Technology. 3rd ed. Academic Press, Wageningen, the Netherlands.
Buriti, F. C., I. A. Castro, and S. M. Saad. 2010. Viability of Lactobacillus acidophilus in synbiotic guava mousses and its survival under in vitro simulated gastrointestinal conditions. Int. J. Food Microbiol. 137:121-129.

Costa, M. P., B. S. Frasao, A. C. O. Silva, M. Q. Freitas, R. M. Franco, and C. A. Conte-Junior. 2015. Cupuassu (Theobroma grandiflorum) pulp, probiotic, and prebiotic: Influence on color, apparent viscosity, and texture of goat milk yogurts. J. Dairy Sci. 98:5995-6003

Cui, B., Y.-M. Lu, C.-P. Tan, G.-Q. Wang, and G.-H. Li. 2014. Effect of cross-linked acetylated starch content on the structure and stability of set yoghurt. Food Hydrocoll. 35:576-582.

Etchepare, M. A. J., C. R. F. Grosso, C. R. de Menezes, E. JacobLopes, G. C. Raddatz, J. S. Barin, and L. Q. Zepka. 2016. Effect of resistant starch (Hi-maize) on the survival of Lactobacillus acidophilus microencapsulated with sodium alginate. J. Funct. Foods 21:321-329.

Hill, C., F. Guarner, G. Reid, G. R. Gibson, D. J. Merenstein, B. Pot, L. Morelli, R. B. Canani, H. J. Flint, and S. Salminen. 2014. Expert consensus document: The International Scientific Association for Probiotics and Prebiotics consensus statement on the scope and appropriate use of the term probiotic. Nat. Rev. Gastroenterol. Hepatol. 11:506-514.

Jana, P., L. Denisa, and V. L'Ubomír. 2015. Suitability of lactic acid bacteria for fermentation of maize and amaranth. J. Food Nutr. Res. 54:354-364.

Jiaojiao, Y. U., M. A. Yafang, D. Wen, Z. Gao, L. I. Wei, W. Tang, and M. Dong. 2015. Effects of fructooligosaccharide and galactooligosaccharide at different concentrations on the quality of fermented milk. Shipin Kexue (Beijing) 36:66-70.

Kett, A. P., V. Chaurin, S. M. Fitzsimons, E. R. Morris, J. A O'Mahony, and M. A. Fenelon. 2013. Influence of milk proteins on the pasting behaviour and microstructural characteristics of waxy maize starch. Food Hydrocoll. 30:661-671.

Lazaridou, A., A. Serafeimidou, C. G. Biliaderis, T. Moschakis, and N. Tzanetakis. 2014. Structure development and acidification kinetics in fermented milk containing oat $\beta$-glucan, a yogurt culture and a probiotic strain. Food Hydrocoll. 39:204-214.

Liu, K., X. Zeng, L. Qiao, X. Li, Y. Yang, C. Dai, A. Hou, and D. Xu. 2014. The sensitivity and significance analysis of parameters in the model of $\mathrm{pH}$ regulation on lactic acid production by Lactobacillus bulgaricus. BMC Bioinformatics 15(Suppl. 13):S5.

Lobato-Calleros, C., C. Ramírez-Santiago, E. Vernon-Carter, and J. Alvarez-Ramirez. 2014. Impact of native and chemically modified starches addition as fat replacers in the viscoelasticity of reducedfat stirred yogurt. J. Food Eng. 131:110-115.

Marafon, A. P., A. Sumi, D. Granato, M. Alcantara, A. Tamime, and M. N. de Oliveira. 2011. Effects of partially replacing skimmed milk powder with dairy ingredients on rheology, sensory profiling, and microstructure of probiotic stirred-type yogurt during cold storage. J. Dairy Sci. 94:5330-5340.

Mishra, S., and H. Mishra. 2013. Effect of synbiotic interaction of fructooligosaccharide and probiotics on the acidification profile, textural and rheological characteristics of fermented soy milk. Food Bioprocess Technol. 6:3166-3176.

Morelli, L. 2014. Yogurt, living cultures, and gut health. Am. J. Clin. Nutr. 99:1248S-1250S.

Pang, Z., H. Deeth, S. Prakash, and N. Bansal. 2016. Development of rheological and sensory properties of combinations of milk proteins and gelling polysaccharides as potential gelatin replacements in the manufacture of stirred acid milk gels and yogurt. J. Food Eng. 169:27-37.

Prasanna, P. H. P., A. S. Grandison, and D. Charalampopoulos. 2014 Bifidobacteria in milk products: An overview of physiological and biochemical properties, exopolysaccharide production, selection criteria of milk products and health benefits. Food Res. Int. $55: 247-262$.

Raigond, P., R. Ezekiel, and B. Raigond. 2015. Resistant starch in food: A review. J. Sci. Food Agric. 95:1968-1978.

Ratnayake, W. S., and D. S. Jackson. 2008. Thermal behavior of resistant starches RS 2, RS 3, and RS 4. J. Food Sci. 73:C356-C366. 
Rezaei, R., M. Khomeiri, M. Kashaninejad, M. Mazaheri-Tehrani, and M. Aalami. 2015. Effect of resistant starch and aging conditions on the physicochemical properties of frozen soy yogurt. J. Food Sci. Technol. 52:8164-8171.

Rohart, A., C. Michon, J. Confiac, and V. Bosc. 2016. Evaluation of ready-to-use SMLS and DWS devices to study acid-induced milk gel changes and syneresis. Dairy Sci. Technol. 96:459-475.

Rosburg, V., T. Boylston, and P. White. 2010. Viability of bifidobacteria strains in yogurt with added oat beta-glucan and corn starch during cold storage. J. Food Sci. 75:C439-C444.

Salvatore, E., V. Mazzarello, A. Pirisi, and M. Pes. 2014. Replacement of fat with long-chain inulin in a fresh cheese made from caprine milk. Int. Dairy J. 34:1-5.

Santos, M., E. Gerbino, E. Tymczyszyn, and A. Gómez-Zavaglia. 2015. Prebiotics as protectants of lactic acid bacteria. Cryobiology $71: 550$.

Senaka Ranadheera, C., C. A. Evans, M. C. Adams, and S. K. Baines. 2012. Probiotic viability and physico-chemical and sensory proper- ties of plain and stirred fruit yogurts made from goat's milk. Food Chem. 135:1411-1418.

Sendra, E., M. E. Sayas-Barberá, J. Fernández-López, and J. A. PérezAlvarez. 2016. Effect of food composition on probiotic bacteria viability. Pages 257-269 in Probiotics, Prebiotics, and Synbiotics. R. R. Watson and V. R. Preedy, ed. Elsevier, Amsterdam, the Netherlands.

Sharafbafi, N., S. M. Tosh, M. Alexander, and M. Corredig. 2014 Phase behaviour, rheological properties, and microstructure of oat $\beta$-glucan-milk mixtures. Food Hydrocoll. 41:274-280.

Titapiccolo, G. I., M. Corredig, and M. Alexander. 2011. Acid coagulation behavior of homogenized milk: Effect of interacting and non-interacting droplets observed by rheology and diffusing wave spectroscopy. Dairy Sci. Technol. 91:185-201.

Zheng, B., Y. Zhang, and H. Zeng. 2016. Structural characteristics and prebiotic effects of lotus seed resistant starch. Pages 195-211 in Probiotics, Prebiotics, and Synbiotics. R. R. Watson and V. R. Preedy, ed. Elsevier, Amsterdam, the Netherlands. 